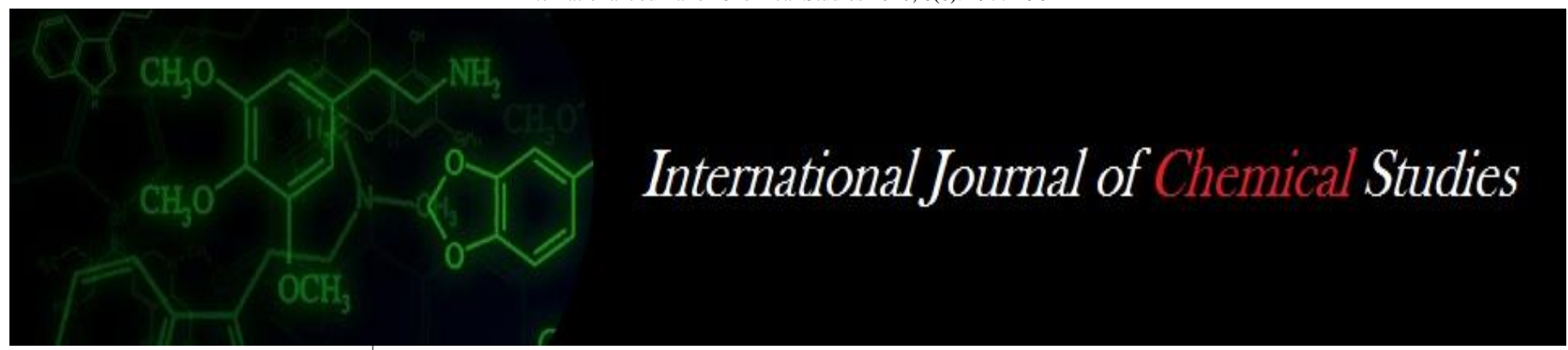

P-ISSN: 2349-8528

E-ISSN: 2321-4902

www.chemijournal.com

IJCS 2020; 8(6): 1977-1982

(C) 2020 IJCS

Received: 04-08-2020

Accepted: 15-10-2020

\section{PN Jagadev}

Department of Plant Breeding and Genetics, Odisha University of Agriculture and Technology, Bhubaneswar, Odisha, India
Corresponding Author: PN Jagadev

Department of Plant Breeding and Genetics, Odisha University of Agriculture and Technology, Bhubaneswar, Odisha, India

\section{Indices based on culm-leaf growth, spike-grain development and relative yield for identification of heat tolerant genotypes in triticale and wheat}

\section{PN Jagadev}

DOI: https://doi.org/10.22271/chemi.2020.v8.i6ab.11057

\section{Abstract}

A study was conducted in a set of 18 triticale and 3 wheat cultivars to identify heat tolerant genotypes under late sowing condition. A numerical taxonomic approach based on culm and leaf growth, spike and grain development and relative yield indices was used and the dendrogram revealed 6 distinct clusters. Two clusters comprising of two triticale (Bulk $\mathrm{MI}_{2}$ and TL 1210) and three wheat genotypes (OW 13, Sagarika and Utkalika) were identified as heat tolerant for use in breeding.

Keywords: Internode growth, grain development, relative yield, heat tolerance, wheat, triticale

\section{Introduction}

Triticale (x Triticosecale Wittmack) is a crop of very limited distribution. Work on various aspects of adaptation and particularly on response to temperature stress in this crop is therefore, much less than the related cereals. Since wheat (Triticum aestivum L. em. Thell.) is one of the parents of triticale and closely related to it, information on adaptation to high temperature in wheat is also relevant. Graphic analysis of internode growth pattern by the ideograph technique (Anderson and Schregardus, 1944) ${ }^{[1]}$ is useful in studying the genetic variation of growth in cereals.. Hence, the present investigation was undertaken to employ the numerical taxonomic approach by the use of indices based on culm (internode) and leaf (leaf sheath) growth, spike and grain development and relative yield for distinguishing the clusters with different growth patterns to select the wheat and triticale genotypes for adaptation to heat stress.

\section{Materials and Methods}

A set of 17 tall triticale (x Triticosecale Wittmack) breeding lines, one released semi-dwarf triticale variety (TL 1210) were taken in the present investigation and three wheat varieties (Sagarika, Utkalika and OW-13) were also included for comparison with the triticale lines. A field trial was undertaken in two dates of sowing; the first $\left(S_{1}\right)$ being the normal sowing (November 15) and the second $\left(S_{2}\right)$ was the late sowing (December 17). The latter date was chosen to subject the plants to heat stress. The magnitude of stress was suggested by the average daily temperatures in two different phases in the two sowings. ( $\mathrm{S}_{1}$ pre-flowering phase: $22.35 \pm 2.17^{\circ} \mathrm{C} ; \mathrm{S}_{1}$ post-flowering phase: $23.91 \pm 1.97^{\circ} \mathrm{C} ; \mathrm{S}_{2}$ pre-flowering phase: 22.83 $\pm 2.28^{\circ} \mathrm{C}$; and $\mathrm{S}_{2}$ post-flowering phase: $\left.26.45 \pm 2.36^{\circ} \mathrm{C}\right)$. Varieties/lines were operational taxonomic units (OTUs) of the numerical taxonomic study indicated in Table 1.

The trial was laid in RBD with three replications. Each line/variety was grown in three rows/replication, each $3 \mathrm{~m}$ long and spaced $25 \mathrm{~cm}$ apart. Fertilizers were applied @ $100 \mathrm{~kg} \mathrm{~N}$, $60 \mathrm{P}_{2} \mathrm{O}_{5}$ and $40 \mathrm{~kg} \mathrm{~K} 2 \mathrm{O}$ per hectare. Data were recorded at maturity on different aspects of vegetative growth (length of successive internodes, $\mathrm{I}_{1}-\mathrm{I}_{6}$, starting with the peduncle as $\mathrm{I}_{1}$ and leaf sheaths, $\mathrm{L}_{1}-\mathrm{L}_{4}$ ). Besides plot yield of grains, data on sampled plants were also recorded for number of spikelets/spike, number of grains/spike, number of grains/spikelet and 100-grain weight. Ten competitive plants were sampled per variety/replication at random at harvest. Numerical taxonomic methods were applied for identifying clusters basing on data of internode growth in $S_{1}$ and $S_{2}$ following Sneath and Sokal (1973) ${ }^{[6]}$. 
The clusters were characterized in terms of response to stress for identifying stress-response types by simple numerical indexing method. A culm growth index (CGI) was calculated for each sowing by summing up individual scores $(0,1$ or 2$)$ for each internode. For assignment of scores, the mean of each variety was examined for its position in the lower middle or upper point of the range of values of all varieties. The deviation index, CGI $\left(\mathrm{S}_{2}\right)$ - CGI $\left(\mathrm{S}_{1}\right)$ was used as the culm growth index of heat tolerance. The leaf growth index (LGI) of heat tolerance was similarly calculated and the sum of these two quantities was called culm and leaf growth (CLG) index of tolerance. Genotypes with positive values were considered tolerant and those with negative and zero values were considered sensitive and moderately tolerant, respectively (Jagadev et al., 1988) ${ }^{[2]}$.

Table 1: Details of triticale and wheat genotypes used in the study

\begin{tabular}{|c|c|c|c|}
\hline Sl. No. in field trial & OTU No. & Name of the culture/ variety & Information about the genotypes \\
\hline 1 & 1 & $\mathrm{Bulk} \mathrm{LI}_{2}$ & Selection under low fertility and two irrigations. \\
\hline 2 & 2 & Bulk LI $_{1}$ & Selection under low fertility and one irrigation. \\
\hline 3 & 3 & Bulk LI ${ }_{0}$ & Selection under low fertility and no irrigation. \\
\hline 4 & 4 & Bulk $\mathrm{MI}_{2}$ & Selection under medium fertility and two irrigations. \\
\hline 5 & 5 & Bulk $\mathrm{HI}_{2}$ & Selection under high fertility and two irrigations. \\
\hline 6 & 6 & Bulk $\mathrm{HI}_{\mathrm{N}}$ & Selection under high fertility and normal irrigation. \\
\hline 7 & 7 & Adv. I & Selection under high fertility and normal irrigation. \\
\hline 8 & 8 & Adv. III & Selection under high fertility and normal irrigation. \\
\hline 9 & 9 & Adv. IV & Selection under high fertility and normal irrigation. \\
\hline 10 & 10 & $\mathrm{HF}$ & Selection under high spike fertility. \\
\hline 11 & - & HF 1 & Died and discarded. \\
\hline 12 & 11 & OW 13 & Wheat variety (OUAT, Bhubaneswar). \\
\hline 13 & 12 & Sagarika & Wheat variety (OUAT, Bhubaneswar). \\
\hline 14 & 13 & GF 1 & Selected for high grain filling. \\
\hline 15 & 14 & GF 1-1a & Selected for grain filling, non-hairy, early, spreading. \\
\hline 16 & 15 & Bulk 1a & Selected from $\mathrm{LI}_{2}$ bulk, hairy, early, medium height. \\
\hline 17 & 16 & Bulk 3 & Selected from $\mathrm{HI}_{2}$ bulk, early, medium height. \\
\hline 18 & 17 & GF $1-1 b$ & Selected for grain filling, non-hairy, early, medium height. \\
\hline 19 & 18 & Bulk 1b & Selected from $\mathrm{LI}_{2}$ bulk, non-hairy, early, tall. \\
\hline 20 & 19 & GF 1-1c & Selected for grain filling, non-hairy, early, medium height. \\
\hline 21 & 20 & TL 1210 & Triticale variety (PAU, Ludhiana). \\
\hline 22 & 21 & Utkalika & Wheat variety (OUAT, Bhubaneswar). \\
\hline- & 22 & - & Average of all the above genotypes. \\
\hline
\end{tabular}

Since the two indices (CGI and LGI) were based on only growth characters, a comparison with an index involving aspects of reproductive development was considered important. Four characters (viz. Number of spikelets/spike, grains per spike and spikelet, 100-grain weight) were used for this index of heat tolerance, known as spike-grain development (SGD) index. For each trait, the degree of reduction in $S_{2}$ (as \% of $S_{1}$ value) was scored $0,1,2$ etc. at 10 $\%$ intervals (e.g., $\mathrm{S}_{2} / \mathrm{S}_{1}$ between $91-100 \%=0$, between $81-90$ $\%=1$ and so on). The scores of all the traits were added to obtain SGD index for each line/variety. The lower the index, the greater the degree of heat tolerance. Index values of 0-5 (lower $1 / 3^{\text {rd }}$ range) was considered as tolerant, 6-10 (middle range) as moderately tolerant and above 10 as sensitive.

Since high temperature in the late sowing invariably caused reduction in yield, the relative $S_{2}$ yield (as $\%$ of $S_{1}$ yield) was taken as a simple and direct measure of response. High and low relative yield (RY) with reference to the grand mean of all the cultivars, would indicate tolerance and sensitivity. Genotypes with above-average yield considered as tolerant, equal/nearing the average yield as moderately tolerant and below-average yield as sensitive.

\section{Results and Discussion}

A numerical taxonomic approach with clustering analysis helped to identify distinct groups or clusters of lines/varieties with similar response to environment or adaptation pattern. Hence, this analysis was undertaken to identify clusters with different growth response to the stress of late sowing and to examine the scope of use of such classification for an evaluation of adaptation to such stress.

The similarity indices estimated on the basis of 22 OTUs ranged from 0.33 (between Utkalika and GF-1-1a) to 0.93 (between Bulk $\mathrm{HI}_{\mathrm{N}}$ and Bulk $\mathrm{LI}_{0}$ and between GF 1-1c and GF 1). The triticales particularly Bulk $\mathrm{LI}_{1}, \mathrm{Bulk} \mathrm{LI}_{2}, \mathrm{Adv}$. IV, Bulk 1a, Bulk 1b and GF 1-1c were highly similar (average similarly of $>90 \%$ ) (Table 2). Cluster analysis based on Gower's coefficients using UPGMA (unweighted pair group method for arithmetic average analysis) classified all the 22 OTUs into 6 clusters, two of which included three wheat varieties (Fig. 1). Cluster 1 contained 9 triticales and the hypothetical average OTU (22), hence called as "average cluster". The composition of clusters of all the genotypes identified from the dendrogram at 85-phenon level, was given in Table 3. The clusters showed distinct culm growth patterns considering both the sowings (Fig. 2). Clusters 3 and 5 clearly showed a different type of growth response in $\mathrm{S}_{2}$ as compared to other 4 clusters. In this the trend of $S_{1}-S_{2}$ growth difference in respect of 3 basal internodes were strikingly less than the same in other clusters. Since this kind of culm growth response accompanied by relatively greater reduction in total height, clusters 3 and 5 were identified as sensitive to high temperature stress. But in clusters 4 and 6 , the trend of $S_{1}-S_{2}$ growth difference in the case of basal internodes being greater than the other clusters, correspondingly difference in total culm length were relatively less, which clearly identified as stress tolerant. The remaining two clusters i.e. 1 and 2 showed an intermediate type of growth response to stress, since cluster 1 represented the average and cluster 2 was closely related to that (Fig. 2). 
Table 2: Similarity matrix of Gower's coefficients for 22 OTUs based on culm (internode) growth in triticale and wheat genotypes

\begin{tabular}{|c|c|c|c|c|c|c|c|c|c|c|c|c|c|c|c|c|c|c|c|c|c|c|}
\hline OTU No. & 1 & 2 & 3 & 4 & 5 & 6 & 7 & 8 & 9 & 10 & 11 & 12 & 13 & 14 & 15 & 16 & 17 & 18 & 19 & 20 & 21 & 22 \\
\hline 1 & 1.00 & & & & & & & & & & & & & & & & & & & & & \\
\hline 2 & 0.87 & 1.00 & & & & & & & & & & & & & & & & & & & & \\
\hline 3 & 0.90 & 0.92 & 1.00 & & & & & & & & & & & & & & & & & & & \\
\hline 4 & 0.80 & 0.76 & 0.81 & 1.00 & & & & & & & & & & & & & & & & & & \\
\hline 5 & 0.88 & 0.86 & 0.89 & 0.77 & 1.00 & & & & & & & & & & & & & & & & & \\
\hline 6 & 0.91 & 0.90 & 0.93 & 0.76 & 0.90 & 1.00 & & & & & & & & & & & & & & & & \\
\hline 7 & 0.79 & 0.76 & 0.73 & 0.66 & 0.78 & 0.75 & 1.00 & & & & & & & & & & & & & & & \\
\hline 8 & 0.87 & 0.87 & 0.83 & 0.79 & 0.82 & 0.84 & 0.85 & 1.00 & & & & & & & & & & & & & & \\
\hline 9 & 0.86 & 0.91 & 0.87 & 0.77 & 0.87 & 0.86 & 0.78 & 0.88 & 1.00 & & & & & & & & & & & & & \\
\hline 10 & 0.84 & 0.89 & 0.87 & 0.71 & 0.82 & 0.88 & 0.79 & 0.87 & 0.85 & 1.00 & & & & & & & & & & & & \\
\hline 11 & 0.79 & 0.76 & 0.83 & 0.72 & 0.77 & 0.82 & 0.62 & 0.72 & 0.72 & 0.74 & 1.00 & & & & & & & & & & & \\
\hline 12 & 0.58 & 0.46 & 0.53 & 0.50 & 0.53 & 0.55 & 0.45 & 0.48 & 0.47 & 0.43 & 0.68 & 1.00 & & & & & & & & & & \\
\hline 13 & 0.91 & 0.88 & 0.89 & 0.75 & 0.88 & 0.91 & 0.78 & 0.86 & 0.89 & 0.85 & 0.79 & 0.57 & 1.00 & & & & & & & & & \\
\hline 14 & 0.70 & 0.71 & 0.67 & 0.66 & 0.66 & 0.67 & 0.85 & 0.79 & 0.75 & 0.73 & 0.53 & 0.38 & 0.72 & 1.00 & & & & & & & & \\
\hline 15 & 0.79 & 0.79 & 0.79 & 0.70 & 0.80 & 0.78 & 0.76 & 0.80 & 0.80 & 0.75 & 0.70 & 0.49 & 0.84 & 0.72 & 1.00 & & & & & & & \\
\hline 16 & 0.86 & 0.83 & 0.83 & 0.80 & 0.80 & 0.81 & 0.79 & 0.89 & 0.84 & 0.79 & 0.76 & 0.57 & 0.82 & 0.75 & 0.73 & 1.00 & & & & & & \\
\hline 17 & 0.82 & 0.81 & 0.77 & 0.76 & 0.78 & 0.79 & 0.84 & 0.89 & 0.85 & 0.79 & 0.64 & \begin{tabular}{|l|l|}
0.46 \\
\end{tabular} & 0.82 & 0.88 & 0.79 & 0.85 & 1.00 & & & & & \\
\hline 18 & 0.85 & 0.83 & 0.86 & 0.76 & 0.88 & 0.85 & 0.77 & 0.82 & 0.84 & 0.80 & 0.77 & 0.53 & 0.86 & 0.69 & 0.90 & 0.77 & 0.80 & 1.00 & & & & \\
\hline 19 & 0.89 & 0.88 & 0.92 & 0.79 & 0.89 & 0.89 & 0.74 & 0.86 & 0.85 & 0.85 & 0.82 & 0.56 & 0.93 & 0.69 & 0.83 & 0.82 & 0.79 & 0.88 & 1.00 & & & \\
\hline 20 & 0.62 & 0.52 & 0.58 & 0.54 & 0.55 & 0.58 & 0.50 & 0.56 & 0.51 & 0.52 & 0.63 & 0.77 & 0.59 & 0.47 & 0.48 & 0.64 & 0.52 & 0.54 & 0.59 & 1.00 & & \\
\hline 21 & 0.53 & 0.42 & 0.49 & 0.45 & 0.48 & 0.50 & 0.36 & 0.44 & 0.42 & 0.38 & 0.62 & 0.78 & 0.52 & 0.33 & 0.44 & 0.52 & 0.42 & 0.48 & 0.52 & 0.81 & 1.00 & \\
\hline 22 & 0.95 & 0.88 & 0.90 & 0.79 & 0.89 & 0.90 & 0.80 & 0.88 & 0.87 & 0.84 & 0.79 & 0.58 & 0.91 & 0.73 & 0.80 & 0.89 & 0.84 & 0.85 & 0.90 & 0.63 & 0.53 & 1.00 \\
\hline
\end{tabular}

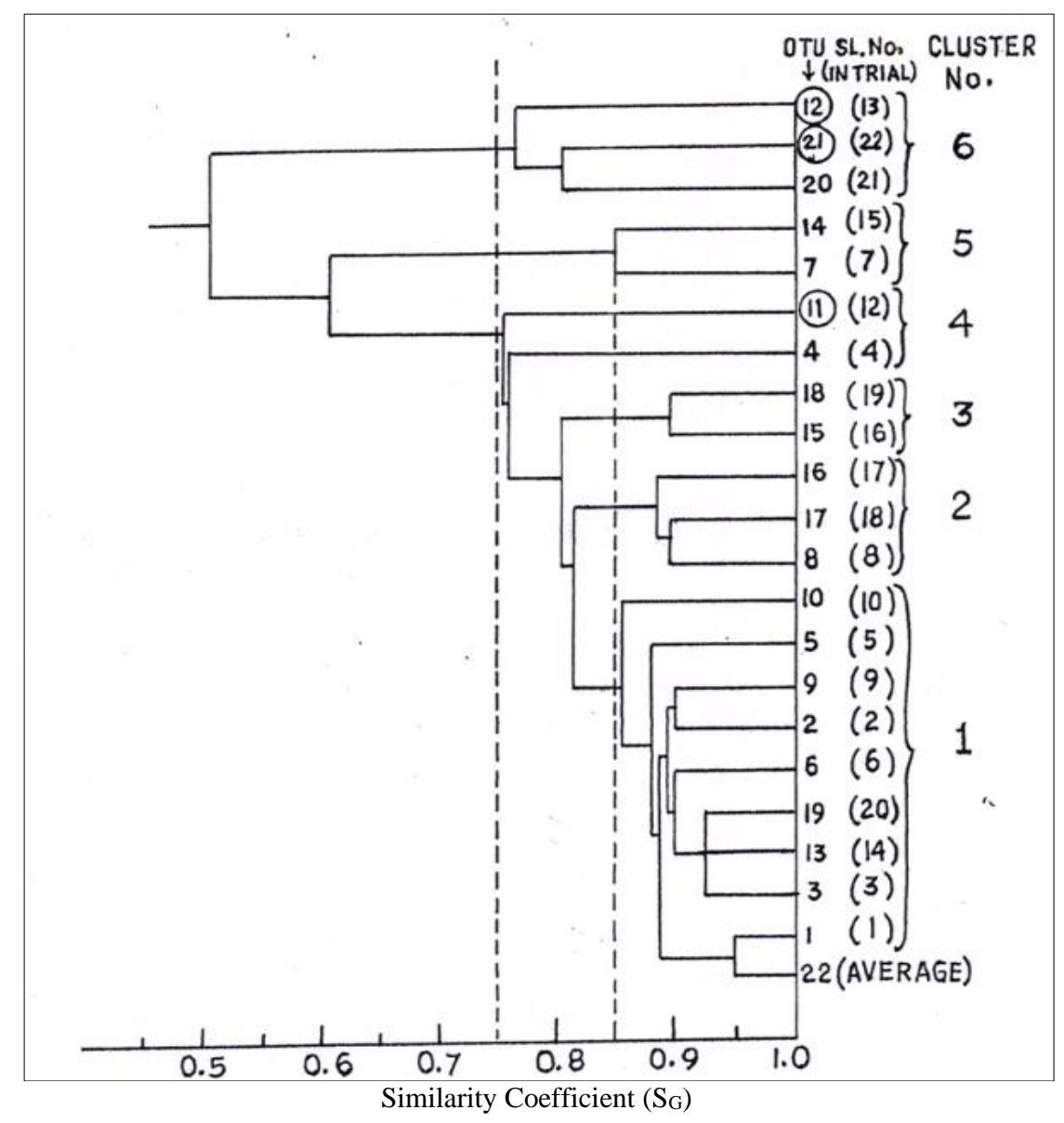

Fig 1: Dendrogram based on similarity coefficients of internode growth in $S_{1}$ and $S_{2}$.

Table 3: Composition of clusters identified from dendrogram at 85-phenon level of triticale and wheat genotypes

\begin{tabular}{|c|c|c|}
\hline Cluster No. & No. of genotypes & Composition of clusters (Sl. Nos. in field trial were given in parentheses) \\
\hline 1 & 9 & 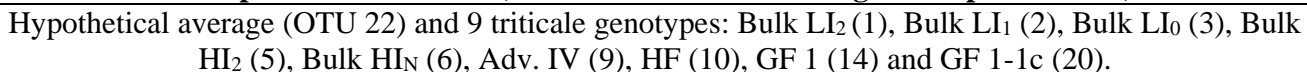 \\
\hline 2 & 3 & 3 triticale genotypes: Adv. III (8), Bulk 3 (17) and GF 1-1b (18) \\
\hline 3 & 2 & 2 tricale genotypes: Bulk 1a (16) and Bulk 1 b (19) \\
\hline 4 & 2 & One triticale genotype : Bulk $\mathrm{MI}_{2}(4)$ and one wheat genotype : OW 13 (12) \\
\hline 5 & 2 & 2 triticale genotypes : Adv. 1 (7) and GF 1-1a (15) \\
\hline 6 & 3 & One triticale genotype : TL 1210 (21) and 2 wheat genotypes : Sagarika (13) and Utkalika (22) \\
\hline
\end{tabular}




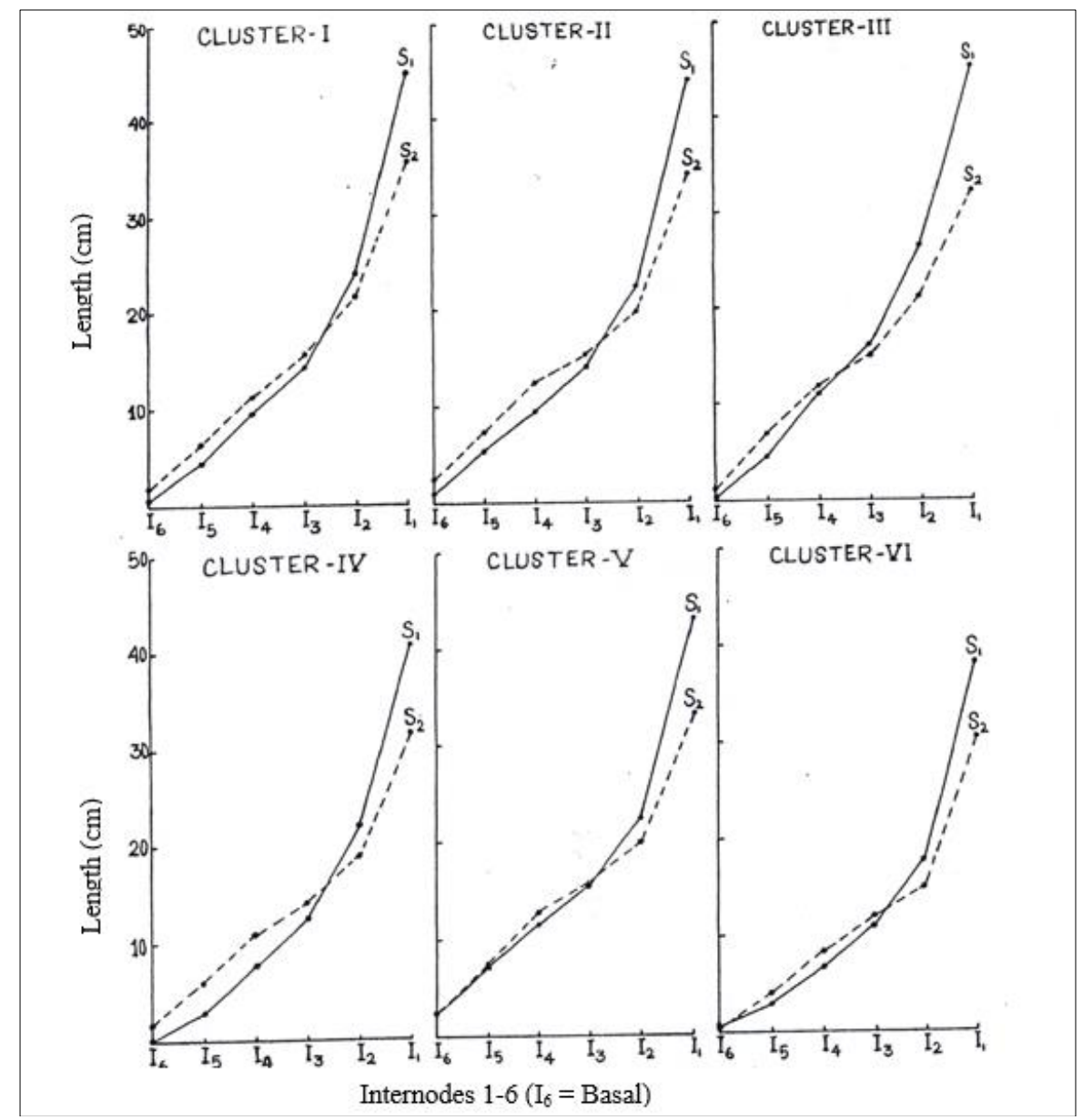

Fig 2: Ideographs of internode growth in different clusters of $S_{1}$ and $S_{2}$

For a meaningful characterization of the clusters, three different indices (viz., CLG, SGD and RY) of heat tolerance were devised. The index values and characterization of the clusters based on CLG (culm and leaf growth) of heat tolerance/sensitivity were presented in Table 4 . The distribution of heat response types, i.e., tolerant (T), moderately tolerant/sensitive (M) and sensitive (S) was presented in a scatter diagram of the two sets of values of growth based sensitivity/tolerance index (Fig. 3). On the basis of the distribution of these broad types of stress (heat) response, the clusters could be characterized as sensitive (Clusters 3 and 5), tolerant (Clusters 4 and 6), moderately tolerant (Cluster 2) and mixed, i.e. T and S (Cluster 1).

Table 4: Culm and leaf growth (CLG) index of heat tolerance and characterization of clusters in triticale and wheat genotypes

\begin{tabular}{|c|c|c|c|c|c|c|}
\hline Cluster No. & Sl. No. in field trial & $\begin{array}{c}\text { Culm growth } \\
\text { index }\end{array}$ & $\begin{array}{c}\text { Leaf growth } \\
\text { index }\end{array}$ & CLG index & $\begin{array}{c}\text { Stress response of } \\
\text { genotype* }\end{array}$ & $\begin{array}{c}\text { Characterization } \\
\text { of cluster }\end{array}$ \\
\hline \multirow{9}{*}{1} & 1 & -1 & -1 & -2 & $\mathrm{~S}$ & \multirow{9}{*}{$\mathrm{T}-\mathrm{S}$} \\
\hline & 2 & +2 & -1 & +1 & $\mathrm{~T}$ & \\
\hline & 3 & +1 & +1 & +2 & $\mathrm{~T}$ & \\
\hline & 5 & 0 & 0 & 0 & $\mathrm{M}$ & \\
\hline & 6 & 0 & -3 & -3 & $\mathrm{~S}$ & \\
\hline & 9 & +2 & 0 & +2 & $\mathrm{~T}$ & \\
\hline & 10 & 0 & -3 & -3 & $\mathrm{~S}$ & \\
\hline & 14 & -1 & -1 & -2 & $\mathrm{~S}$ & \\
\hline & 20 & -2 & 0 & -2 & $\mathrm{~S}$ & \\
\hline \multirow{3}{*}{2} & 8 & 0 & 0 & 0 & $\mathrm{M}$ & \multirow{3}{*}{ M } \\
\hline & 17 & +2 & +4 & +6 & $\mathrm{~T}$ & \\
\hline & 18 & -1 & +1 & 0 & $\mathrm{M}$ & \\
\hline \multirow{2}{*}{3} & 16 & -3 & 0 & -3 & $\mathrm{~S}$ & \multirow{2}{*}{ S } \\
\hline & 19 & -2 & -2 & -4 & $S$ & \\
\hline \multirow{2}{*}{4} & 4 & +1 & +1 & +2 & $\mathrm{~T}$ & \multirow{2}{*}{$\mathrm{T}$} \\
\hline & 12 & +4 & 0 & +4 & $\mathrm{~T}$ & \\
\hline \multirow{2}{*}{5} & 7 & -2 & 0 & -2 & $\mathrm{~S}$ & \multirow{2}{*}{$S$} \\
\hline & 15 & 0 & 0 & 0 & $\mathrm{M}$ & \\
\hline \multirow{3}{*}{6} & 13 & +2 & 0 & +2 & $\mathrm{~T}$ & \multirow{3}{*}{$\mathrm{T}$} \\
\hline & 21 & +1 & +1 & +2 & $\mathrm{~T}$ & \\
\hline & 22 & 0 & 0 & 0 & $\mathrm{M}$ & \\
\hline
\end{tabular}




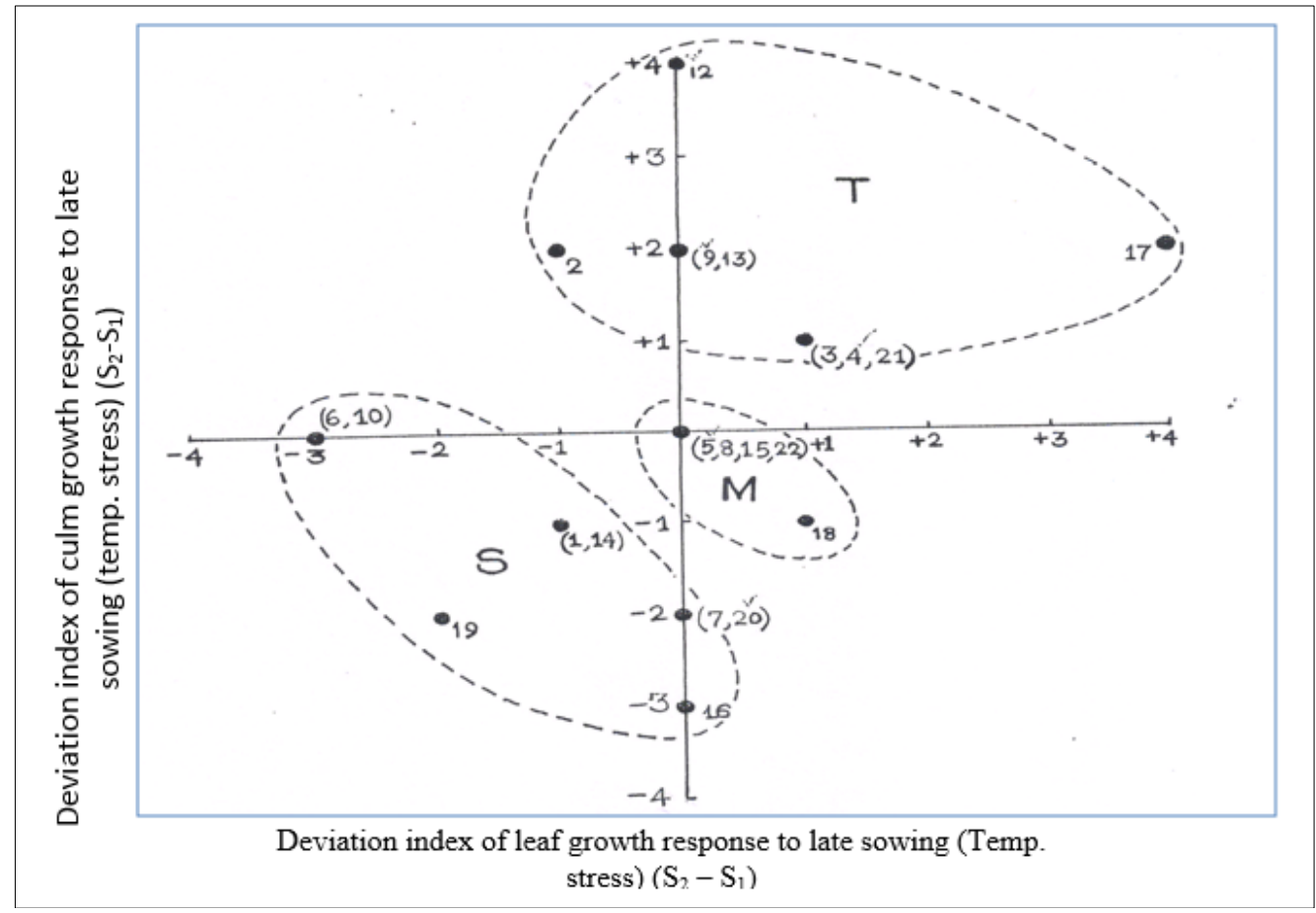

Fig 3: Scatter plot of deviation indices for culm and leaf growth response to stress

The index values and characterization of clusters based on SGD (spike and grain development) of heat tolerance were presented in Table 5 and the following points were worth nothing.

1. The two clusters (Cl. 3 and 5) identified as sensitive (S) on the basis of growth (CLG) index showed sensitive or moderate type of response to stress as judged by the SGD index

2. Out of the two clusters ( $\mathrm{Cl} .4$ and 6), similarly identified by the growth index as tolerant $(\mathrm{T})$, one $(\mathrm{Cl} .6)$ also appeared to be tolerant by the SGD criterion and the other (Cl. 4) showed moderate type of response.

3. Considering these two pairs of extreme clusters with similar growth response to stress, the SGD index also helped to identify those as the extremes, when the average SGD index of paired clusters considered.

4. The remaining closely related cluster pair (Cl. 1 and 2) would show all the types of stress-response as also noted earlier for growth. However, the average response in each case and for the pair was of the moderate (M) type.

5. The three cluster pairs were characterized as $\mathrm{T}$ or $\mathrm{M}$ or $\mathrm{S}$ by both measures of heat sensitivity/tolerance. The average SGD index values of those three cluster pairs were 3.7 for $\mathrm{T}, 6.3$ for $\mathrm{M}$ and 10.3 for $\mathrm{S}$ response classes.

6. Thus growth based characterization of clusters was at least broadly comparable to the response categories based on later development (SGD).

Table 5: Spike and grain development (SGD) index of heat sensitivity and characterization of clusters grouped according to growth based response

\begin{tabular}{|c|c|c|c|c|c|c|c|}
\hline $\begin{array}{l}\text { Cluster No. (type of } \\
\text { growth based response) }\end{array}$ & $\begin{array}{l}\text { Sl. No. in } \\
\text { field trial }\end{array}$ & $\begin{array}{c}\text { SGD index } \\
\text { value }\end{array}$ & $\begin{array}{c}\text { Type of stress } \\
\text { response }\end{array}$ & \begin{tabular}{|c|} 
Average SGD \\
index of cluster
\end{tabular} & \begin{tabular}{|c|}
$\begin{array}{c}\text { Characterization } \\
\text { of cluster }\end{array}$ \\
\end{tabular} & $\begin{array}{c}\text { Average SGD index } \\
\text { of paired clusters }\end{array}$ & \begin{tabular}{|c|}
$\begin{array}{c}\text { Characterization } \\
\text { of paired clusters }\end{array}$ \\
\end{tabular} \\
\hline \multirow{9}{*}{$\begin{array}{c}1 \\
(\mathrm{~T}-\mathrm{S})\end{array}$} & 1 & 8 & $\mathrm{M}$ & \multirow{9}{*}{5.6} & \multirow{9}{*}{$\mathrm{M}$} & \multirow{12}{*}{6.3} & \multirow[t]{12}{*}{$\begin{array}{ll} \\
\end{array}$} \\
\hline & 2 & 3 & $\mathrm{~T}$ & & & & \\
\hline & 3 & 1 & $\mathrm{~T}$ & & & & \\
\hline & 5 & 5 & $\mathrm{~T}$ & & & & \\
\hline & 6 & 7 & $\mathrm{M}$ & & & & \\
\hline & 9 & 3 & $\mathrm{~T}$ & & & & \\
\hline & 10 & 6 & $\mathrm{M}$ & & & & \\
\hline & 14 & 5 & $\mathrm{~T}$ & & & & \\
\hline & 20 & 12 & $S$ & & & & \\
\hline \multirow{3}{*}{$\begin{array}{c}2 \\
(\mathrm{M})\end{array}$} & 8 & 11 & $S$ & \multirow{3}{*}{8.3} & \multirow{3}{*}{ M } & & \\
\hline & 17 & 7 & $\mathrm{M}$ & & & & \\
\hline & 18 & 7 & $\mathrm{M}$ & & & & \\
\hline 3 & 16 & 15 & $S$ & 120 & $\mathrm{~S}$ & \multirow{4}{*}{10.3} & \multirow{4}{*}{$S$} \\
\hline$(\mathrm{S})$ & 19 & 9 & $\mathrm{M}$ & 12.0 & $\mathrm{~S}$ & & \\
\hline 5 & 7 & 9 & $\mathrm{M}$ & \multirow{2}{*}{8.5} & \multirow{2}{*}{ M } & & \\
\hline (S) & 15 & 8 & $\mathrm{M}$ & & & & \\
\hline 4 & 4 & 5 & $\mathrm{~T}$ & & & \multirow{5}{*}{3.7} & \multirow{5}{*}{$\mathrm{T}$} \\
\hline$(\mathrm{T})$ & 12 & 7 & $\mathrm{M}$ & 6.0 & $\mathrm{M}$ & & \\
\hline \multirow{3}{*}{$\begin{array}{c}6 \\
(\mathrm{~T})\end{array}$} & 13 & 3 & $\mathrm{~T}$ & \multirow{3}{*}{1.3} & \multirow{3}{*}{$\mathrm{T}$} & & \\
\hline & 21 & 1 & $\mathrm{~T}$ & & & & \\
\hline & 22 & 0 & $\mathrm{~T}$ & & & & \\
\hline
\end{tabular}

$* \mathrm{~S}=$ Sensitive, $\mathrm{T}=$ Tolerant, $\mathrm{M}=$ Moderately tolerant 
The index values and characterization of clusters based on relative $S_{2}$ yield (RY) as a measure of heat tolerance were presented in Table 6 and the following inferences were established.

1. The sensitive cluster pair (Cl. 3 and 5) were evidently heat sensitive (S) by this RY criterion also.

2. In the case of 'tolerant' cluster pair ( $\mathrm{Cl} .4$ and 6$)$, one $(\mathrm{Cl}$.
4) was evidently tolerant by the RY measure, but considering the RY index values for the paired clusters, the cluster pair was characterized as 'tolerant' $(\mathrm{T})$.

3. In the remaining cluster pair ( $\mathrm{Cl} .1$ and 2$)$, the average cluster (Cl.1) was tolerant according to RY criterion, but considering the RY index values for paired clusters, the cluster pair was characterized as moderately tolerant (M).

Table 6: Relative $S_{2}$ yield (RY) index of heat sensitivity and characterization of clusters grouped according to growth based response

\begin{tabular}{|c|c|c|c|c|c|c|c|c|c|}
\hline \multirow{2}{*}{$\begin{array}{c}\text { Cluster } \\
\text { No. }\end{array}$} & \multirow{2}{*}{$\begin{array}{l}\text { Sl. No. in } \\
\text { field trial }\end{array}$} & \multicolumn{2}{|c|}{ Yield (kg/ha) } & \multirow{2}{*}{$\begin{array}{c}\text { Relative } S_{2} \text { yield } \\
\text { index (as \% of } S_{1} \\
\text { yield) }\end{array}$} & \multirow{2}{*}{$\begin{array}{l}\text { Type of } \\
\text { stress } \\
\text { response }\end{array}$} & \multirow{2}{*}{\begin{tabular}{|c|} 
Average RY \\
index of \\
cluster
\end{tabular}} & \multirow{2}{*}{$\begin{array}{c}\text { Characterizati } \\
\text { on of cluster }\end{array}$} & \multirow{2}{*}{\begin{tabular}{|c|} 
Average RY \\
index of \\
paired cluster
\end{tabular}} & \multirow{2}{*}{\begin{tabular}{|c|} 
Characterizat \\
ion of paired \\
clusters
\end{tabular}} \\
\hline & & $\mathbf{S}_{1}$ & $\mathbf{S}_{2}$ & & & & & & \\
\hline \multirow{9}{*}{1} & 1 & 918.51 & 533.33 & 58.1 & $\mathrm{~T}$ & \multirow{9}{*}{58.5} & \multirow{9}{*}{$\mathrm{T}$} & \multirow{12}{*}{56.1} & \multirow{12}{*}{ M } \\
\hline & 2 & 888.88 & 414.81 & 46.7 & $S$ & & & & \\
\hline & 3 & 770.36 & 562.96 & 73.4 & $\mathrm{~T}$ & & & & \\
\hline & 5 & 1274.06 & 740.74 & 58.1 & $\mathrm{~T}$ & & & & \\
\hline & 6 & 1037.03 & 444.44 & 42.9 & $S$ & & & & \\
\hline & 9 & 1037.03 & 681.47 & 65.7 & $\mathrm{~T}$ & & & & \\
\hline & 10 & 881.48 & 592.59 & 67.2 & $\mathrm{~T}$ & & & & \\
\hline & 14 & 1066.66 & 385.18 & 36.1 & $\mathrm{~S}$ & & & & \\
\hline & 20 & 814.81 & 637.03 & 78.2 & $\mathrm{~T}$ & & & & \\
\hline \multirow{3}{*}{2} & 8 & 962.96 & 562.96 & 58.5 & $\mathrm{~T}$ & \multirow{3}{*}{53.7} & \multirow{3}{*}{$S$} & & \\
\hline & 17 & 1081.48 & 533.33 & 49.3 & $S$ & & & & \\
\hline & 18 & 918.51 & 488.88 & 53.2 & $\mathrm{~S}$ & & & & \\
\hline \multirow{2}{*}{3} & 16 & 674.07 & 237.03 & 35.2 & $\mathrm{~S}$ & \multirow{2}{*}{54.2} & \multirow{2}{*}{$S$} & \multirow{4}{*}{51.2} & \multirow{4}{*}{$\mathrm{S}$} \\
\hline & 19 & 1051.85 & 770.36 & 73.2 & $\mathrm{~T}$ & & & & \\
\hline \multirow{2}{*}{5} & 7 & 1259.25 & 503.70 & 40.0 & $\mathrm{~S}$ & \multirow{2}{*}{48.1} & \multirow{2}{*}{$S$} & & \\
\hline & 15 & 1318.51 & 740.73 & 56.2 & $\mathrm{M}$ & & & & \\
\hline \multirow{2}{*}{4} & 4 & 1185.18 & 740.74 & 62.5 & $\mathrm{~T}$ & \multirow{2}{*}{65.0} & \multirow{2}{*}{$\mathrm{T}$} & \multirow{5}{*}{57.4} & \multirow{5}{*}{$\mathrm{T}$} \\
\hline & 12 & 1185.18 & 799.99 & 67.5 & $\mathrm{~T}$ & & & & \\
\hline \multirow{3}{*}{6} & 13 & 1259.25 & 562.96 & 44.7 & $S$ & \multirow{3}{*}{49.7} & & & \\
\hline & 21 & 1007.40 & 607.40 & 60.3 & $\mathrm{~T}$ & & S & & \\
\hline & 22 & 1274.07 & 562.96 & 44.2 & $S$ & & & & \\
\hline & Grand Mea & of all clus & & 55.8 & & & & & \\
\hline
\end{tabular}

*S=Sensitive, $\mathrm{T}=$ Tolerant, $\mathrm{M}=$ moderately tolerant

Hence, the numerical taxonomic analysis based on culm-leaf growth (Jagadev and Sinha, 2006, Sinha and Jagadev, 2003) $[3,5]$, spike-grain development and relative $S_{2}$ yield indices was found to be useful in selection of breeding lines of triticale and wheat for adaptation to high temperature stress in conformity with the findings of Mohapatra et al. (1995) [4] and the two clusters ( $\mathrm{Cl} .4$ and 6), which included two triticale genotypes (Bulk $\mathrm{MI}_{2}$ and $\mathrm{T}$ 1210) and three wheat varieties (Sagarika, Utkalika and OW 13) were identified as heat tolerant.

\section{References}

1. Anderson E, Schregardus D. A method for recording and analyzing variations of internode patterns. Ann. Mo. Bot. Gdn 1944;31:241-247.

2. Jagadev PN, Sinha SK, Mohapatra BK. Index based on internode growth as an aid to selection for high temperature tolerance in wheat and triticale. Plant Sci. Res 1988;10:69-74.

3. Jagadev PN, Sinha SK. Adaptation to temperature stress in triticale and wheat: a numerical taxonomic approach. Proc. 10 ${ }^{\text {th }}$ Orissa Bigyan Cong. (Nov.4-5) 2006, 94-97.

4. Mohapatra KC, Mishra CHP, Acharya B. Clustering of rice mutants by different methods of analysis. Indian $\mathbf{J}$ Genet 1995;55:138-147.

5. Sinha SK, Jagadev PN. A taxometric and semigraphic study of variation in internode growth pattern of triticale and wheat. Proc. $7^{\text {th }}$ Orissa Bigyan Cong. (Nov. 9) 2003, 93-97.
6. Sneath PHA, Sokal RR. Numerical Taxonomy. W.H. Freeman and Co, San Francisco 1973, 228-235.sss 